\title{
Audience Management Practices of Live Streamers on Twitch
}

\author{
Donghee Yvette Wohn \\ wohn@njit.edu \\ New Jersey Institute of Technology
}

\author{
Guo Freeman \\ guof@clemson.edu \\ Clemson University
}

\begin{abstract}
Live streaming is a unique medium that merges different layers of communication by facilitating individual, group, and mass communication simultaneously. Streamers who broadcast themselves on live streaming platforms such as Twitch are their own media entity and have the challenge of having to manage interactions with many different types of online audiences beyond the translucent platform interfaces. Through qualitative interviews with 25 Twitch streamers, in this paper we share streamers' practices of discovering audience composition, categorizing audience groups, and developing appropriate mechanisms to interact with them despite geographical, technological, and temporal limitations. We discuss streamers' appropriation of real-time signals provided by these platforms as sources of information, and their dependence on both technology and voluntary human labor to scale their media entity. We conclude with design recommendations for streaming platforms to provide streamer-centric tools for audience management, especially for knowledge discovery and growth management.
\end{abstract}

\section{CCS CONCEPTS}

- Human-centered computing $\rightarrow$ Empirical studies in collaborative and social computing.

\section{KEYWORDS}

audience management; live streaming; self-presentation; Twitch

\section{ACM Reference Format:}

Donghee Yvette Wohn and Guo Freeman. 2020. Audience Management Practices of Live Streamers on Twitch. In ACM International Conference on Interactive Media Experiences (IMX '20), fune 17-19, 2020, Cornella, Barcelona, Spain. ACM, New York, NY, USA, 11 pages. https://doi.org/10.1145/3391614. 3393653

\section{INTRODUCTION}

Online live streaming and viewing have grown exponentially over the last ten years. According to TechJury, $80 \%$ of consumers now prefer to watch live videos from a brand rather than read a blog, and $63 \%$ of millennials aged 18- 34 are creating and/or viewing live streams regularly [2]. Industry analysts expect live streaming to account for $82 \%$ of all internet traffic by 2020 [1].

Of all the live streaming platforms, Twitch.tv is considered one of the biggest and most popular. It enjoys more than 15 million unique

Permission to make digital or hard copies of part or all of this work for personal or classroom use is granted without fee provided that copies are not made or distributed for profit or commercial advantage and that copies bear this notice and the full citation on the first page. Copyrights for third-party components of this work must be honored For all other uses, contact the owner/author(s).

IMX '20, June 17-19, 2020, Cornella, Barcelona, Spain

(c) 2020 Copyright held by the owner/author(s).

ACM ISBN 978-1-4503-7976-2/20/06.

https://doi.org/10.1145/3391614.3393653 daily visitors, each spending an average of 95 minutes watching live gaming and other content [2]. Between January and May of 2019, there were 4.2 million monthly streamers, and a cumulative 313 billion minutes watched by viewers [3]. As such a significant number of users are viewing content in live-streams, we focus this paper on one of the most significant reasons why live streaming has grown so immensely: the content creators who continue to create attractive content.

These Twitch content creators are responsible for conceptualizing, writing, producing, directing, editing, and performing their content. While juggling these multiple hats, the content creators also have to cultivate, track, manage, engage, and satisfy diverse online audiences. Consequently, these tasks pose a mental and emotional capacity (i.e., bandwidth) challenge because the streamers may not have extra hands (e.g., production crew, managers) who can help with the content creation and/or audience management (the exception being highly successful "celebrity" streamers who are able to hire staff). Due to a limited bandwidth, the streamers try to do their best at managing these different tasks. Since these performance are live, there is an extra layer of pressure to perform these multiple jobs well simultaneously and in a public facing mode. Tools that may support any part of such endeavors are welcomed by the streamers and have the potential to improve the user experience of not just the streamers but consequently the stream and eventually viewer experiences indirectly.

Across these multiple tasks, this paper focuses on the "audience management" aspect of streaming. On the one hand, limited resources and tools have been designed to alleviate the challenges faced by streamers to manage the exponentially growing crowds of viewers. On the other hand, existing research has primarily focused on the viewers or the content production aspect of streaming. Furthermore, while some work has been done from the perspective of audience management on social media platforms (e.g., Facebook, Google+, Twitter, and Reddit) [4, 18, 19, 21, 24, 25, 27], to the best of our knowledge, limited work has focused on audience management during a performative live stream. This open space is worthy of investigation because the real time and high-fidelity nature of interactions that take place on live streaming platforms, which affords streamers less bandwidth than asynchronous social media platforms.

The goal of this paper is to contribute towards $\mathrm{HCI} / \mathrm{CSCW}$ knowledge on how the streamers conceptualize their audiences, the existing mental models of these streamers, challenges faced by them to manage their audience, and how future live streaming tools may benefit from better design to alleviate these challenges. The paper begins by providing background on theories of performance and audience management as well as live streaming, followed by the qualitative method of collecting data from 25 streamers, results from these interviews, and a discussion of our findings with specific design implications. 


\section{BACKGROUND}

\subsection{Performance and Audience Management Online}

In his classic book The Presentation of Self in Everyday Life, Goffman [11] used the metaphor of theatrical performance to highlight the performative and characteristic structure of society. His main argument is that human interaction happens between the "front stage" and the "backstage," as well as between performers and audiences. Goffman's theory offers a conceptual framework that is often used in social sciences to study people's self-presentation online and offline: self-identity is constructed in a collective and interactive process within different social settings; in doing so, it is important for performers (i.e., who endeavor to construct their self-identities) to identify audiences so as to adjust their performance.

Such theories of performance and audience management have also been introduced to the field of computing. Many computermediated communication scholars and $\mathrm{HCI} / \mathrm{CSCW}$ researchers have explored the interplay among people's online presentations, their perceptions of others' presentations, and the affordances of specific sociotechnical systems. In particular, extensive research has been done to emphasize the role of social media profiles as a form of customized, audience-oriented self-presentation. In contrast to traditional self-presentation offline, social media not only offer novel tools and features for self-presentation but also introduce new challenges to understand self-presentation behaviors [10]. Hogan [16] argued that self-presentation on social media platforms consists of two parts: 1) performances, which take place in synchronous "situations" (referring to the notions of front and back stages); and 2) artifacts, which take place in asynchronous"exhibitions" (referring to technological affordances such as status updates, chatting, and uploading photos). DeVito [10] proposed an affordance-based approach to self-presentation on social media platforms, which includes Self, Other Actors, and The Audience.

Some others especially focus on mechanisms and social outcomes of audience management in constructing self-presentation online. One important concept here is the "imagined audience"someone's mental conceptualization of the people with whom they are communicating [24]. Litt and Hargittai [25] highlighted that because users often interacted with large diverse audiences, they coped by envisioning either very broad abstract imagined audiences or more targeted specific imagined audiences, who composed of personal ties, professional ties, communal ties, and/or phantasmal ties. Focusing on information sharing, Kairam et al. explored how users of Google+, a social networking service that focuses on using "Circles" to group contacts, organize and select audiences for shared content [18]. Their findings highlight that Google+ users tended to selectively and separately share content based on different life facets strength of ties, and topical interests; and that in particular, privacy, relevance, social norms, and content distributions were four primary factors when choosing audiences for content [18].

In summary, existing studies have pointed to the importance of studying how users of social media platforms manage the online representations that they create, the information that they share, and the audience whom they interact with. However, they seem to mostly focus on asynchronous social media platforms and emphasize the importance of understanding affordances [10]. How would new and emerging sociotechnical systems with different affordances (e.g., real time, high fidelity, and multi-modality) shape these user-audience dynamics?

\subsection{Audience Management in Live Streaming}

In this paper, our goal is to build upon these existing studies on audience perception and management while leveraging the unique technological affordances of live streaming platforms. In doing so, we especially focus on streamers' audience management practices on Twitch, one of the largest live streaming platforms.

On Twitch, anyone can create an account and broadcast themselves for free. Originally, the streaming content was limited to gaming, but in recent years has become more diverse. Researchers have found that people who stream do so with a performative element [23, 26, 28, 35, 42] that can range from extremely personal to strategic [34]. Pellicone et al. [28] described streaming as a "cultural production" and that streamers must display a distinct identity through their performance to create an attractive persona for viewers, which differs from other streaming services such as mobile videochat, that facilitate more interpersonal interactions [33].

Broadcasting, however, is not the only key feature. Another important affordance of Twitch, which is also true of most live streaming platforms (e.g.,[12, 22]), is a live chat function. This chat function is a text-based live commenting feature much like Internet Relay Chat (IRC) of the early days of the Internet and is displayed on the screen next to the live video, such that viewers can interact with each other and the person who is broadcaster, the streamer.

Because of the social interactions that are facilitated in this medium, much of the research from the perspective of the viewer describes how the social engagement motivations draw in viewer participation (e.g., $[13,15,20])$. Hamilton et al. [13] found that much like other types of social media, people were forming communities of various sizes. They described how the formation of communities were based on shared identities that corresponded to the content of the streams and that the growth of these communities were organic in nature. Hilvert-Bruce et al. [15] also found that meeting new people, social interaction, and sense of community were some of the reasons people watched live streams, and that those who preferred smaller streams were more fueled by those social opportunities than those who preferred the larger streams. In many aspects, the formation of communities in live streaming and the activities and motivations of those who participate are not very different from online communities that were formed in the 80s and 90s [31, 41].

While "community" implies a group effort, many forms of live streaming are unique in that people do not just gather around a content, they are gathering around a person producing the content $[7,35]$, and this creates a unique community structure where not all users in the community are the same, and the interactions that take place between viewers and the relationships that form between viewers are not necessarily the same as that between viewers and the streamer [37]. Even in specialized contexts, such as shopping [6, 7], art [29], or live coding [9], viewers cited interaction with streamers as being a main motivation of participation. Moreover, the nature of how viewers feel about the streamer can be parasocial in nature [17, 39], meaning that viewers can have an emotional 
attachment to the streamer that is not necessarily reciprocated to the same intensity by the streamer [37].

We know that streamers perceive content delivery as a performative act $[23,28]$. Yet less is known regarding how they manage that performance in terms of audience interaction. For example, viewers are developing feelings of closeness and intimacy with streamers $[39,42]-$ but how do streamers facilitate this intimacy when they are broadcasting to strangers online? We thus asked three broad research questions that informed our inquiry:

RQ1: What are streamers' perceived audience?

RQ2: How do streamers learn about their audience?

RQ3: How do streamers manage interactions with different audiences?

\section{METHODOLOGY}

To answer our research questions, we decided to conduct semistructured in-depth interviews to understand the nuances and context of streamers' responses. For RQ1 (what are streamers' perceived audience), we asked interview questions such as "When you are streaming, who do you have in mind" and "How would you characterize your viewers?". For RQ2 (how do streamers learn about their audience), our questions included, among others, "How do you find out who the viewers are?" and "Who do you think watches you?". For RQ3 (how do streamers manage interactions with different audiences?), related questions included "How do you interact with viewers?" and "How do you decide who to respond to?" The interview protocol was reviewed and approved by IRB.

To recruit participants, we attended TwitchCon, a convention for streamers, to recruit Twitch streamers in person. We also recruited via Twitter and through personal contacts. As a result, 25 interviews were conducted over four months through phone or Discord audio. Interviews were audio recorded and transcribed prior to analysis.

All participants are English-speakers from North America or the U.K. Of the 25 participants, 11 self-identified as women and 14 as men. Though the proportion of male streamers is much higher on Twitch, we purposefully endeavored to recruit a sample of balanced gender distribution. Of the participants who volunteered to disclose their race, 13 self-identified as Caucasian, three as African American, three as Hispanic, one as Asian, one as Pacific Islander, and two as mixed race. They were diverse also in terms of age, ranging from 20 to 52 . They also had a wide range of followers - from 119 to 187,664. Table 1 summarizes details of participants.

We then used an empirical, in-depth qualitative analysis of the collected data [32] to explore streamers' practices to identify, manage, and interact with their audiences. Our coding and analytical procedures focused on three directions: 1) we closely read through the interview data to acquire a sense of the whole picture as regards how streamers approached their audience and collectively identified thematic topics and common features in the data (e.g., perceptions, categories, and interaction strategies) for further analysis; 2) we carefully examined and reviewed the thematic topics and developed sub-themes; 3 ) we collaborated in an iterative coding process to discuss, combine, and refine themes and features to generate a rich description synthesizing streamers' audience management practices. In order to extract the themes, we first went through the protocol and identified questions that we thought would have answers closely associated with the research questions. We then took participants' answers to these questions and created units of analysis based on the main thought, and reorganized the answers that corresponded to each of the research question before starting the coding process. The coding and thematic identification practice was similar to that used in affinity diagrams, but we did it digitally through a spreadsheet where each row represented a quote that contained one unit of analysis, and the quotes were then clustered into groups that were color-coded using the cell-shade tool. We then looked that the quotes that we had grouped and discussed what the name of that cluster should be; we originally had 39 themes, some of which we merged or separated into smaller themes, resulting in 14 themes across three research questions.

\section{RESULTS}

Here, we present our findings regarding how streamers discovered, identified, and categorized their audience in their streaming practices as well as their mechanisms and strategies to interact with them. Specifically, we divide our findings into three parts: the perceived audience; strategies to discover who the audiences are; and methods for managing interactions with various audiences.

\subsection{Perceived Audience}

Our first research question investigates the concept of audience from the streamers' perspective: who do they think about when they were streaming? In understanding their audience, we found that streamers created mental categories of their viewers. These categories were not necessarily mutually exclusive but illustrated how streamers conceptualized their viewers. A total of six categories of viewers emerged in our data. Two related to the social meanings that viewers hold to streamers (i.e., family, supporter) and four concerned about streamers' perceptions of viewer characteristics (i.e., people of same social group, people interested in the content or of a specific demographic, lurkers, and trolls).

4.1.1 Family/Community. Some streamers were very close to their viewers, calling them "family," "second family," or "my community." This did not appear to correlate to the number of viewers, but was rather related to the attitude and personality of the streamer. For example, P1 (female, 20, White) described, "I call them 'family' on the stream. It's not a very rigid boundary like a content-creator-viewer boundary or that kind of thing. Um because here is somebody who stumbled on your stream and enjoys your art and wants to spend time with you and is spending an intimate amount of time with you, day after day on your streams. To me, that's friendship."

According to $\mathrm{P} 1$, it was clear that viewers were more than merely online strangers to her due to the "intimate amount of time" that they spent with her. For her, such significant time investment blurred the boundaries among content, creator, and viewer and led to the emergence of online "friendship." To further explain such a friendship, P1 went on to explain that watching a streamer was not the same as watching an actor in a movie. P18 (female, 24, White/Black) echoed this view by describing how she developed emotional bonds through sharing intimate personal troubles: "A lot of us struggle with anxiety which is something that I'm very open about." For streamers like P18, they seemed to feel comfortable to share intimate feelings and stories with their viewers, considering them people whom they 
Table 1: Demographic information of interviewees

\begin{tabular}{cccccc}
\hline Participant ID & Gender & Age & Race & No. of Followers & Streaming Content \\
\hline P1 & Female & 20 & White & 3,622 & First-person shooters \\
P2 & Male & 20 s & Hispanic & 3,983 & Creative \\
P3 & Female & 41 & White & 2,116 & Creative \\
P4 & Male & 34 & White & 37,187 & Tabletop role-playing games \\
P5 & Female & 25 & White & 15,333 & Pokemon games \\
P6 & Female & 19 & Asian & 6,153 & Art/Games \\
P7 & Male & N/A & N/A & 192 & Games \\
P8 & Male & N/A & Mixed & 569 & Art/Games \\
P9 & Male & 26 & White & 239 & Games \\
P10 & Male & 25 & White & 532 & Visual novels, Games \\
P11 & Male & 28 & Hispanic & 119 & Games \\
P12 & Female & 31 & Pacific Islander & 515 & Art/Games \\
P13 & Female & 31 & White & 457 & Games \\
P14 & Male & 40 s & Black & 484 & Games \\
P15 & Female & 52 & White & 933 & Games \\
P16 & Male & 37 & Black & 398 & Games \\
P17 & Male & 34 & White & 1,858 & Boardgames \\
P18 & Female & 24 & White/Black & 519 & Cooking/Games \\
P19 & Female & N/A & White & 18,989 & Music/Games \\
P20 & Male & 29 & N/A & 150 & Games \\
P21 & Male & 27 & White & 54,267 & Mobile Games \\
P22 & Male & N/A & N/A & 477 & Games \\
P23 & Female & 44 & Black & 3,623 & Games \\
P24 & Female & 30 & Hispanic & 187,664 & Games/Beauty/Creative \\
P25 & Male & N/A & White & 481 & Games \\
\hline
\end{tabular}

could trust as family or friends rather than merely viewers of their created content.

4.1.2 Supporters/ Fans. Streamers also had a mental group that they labeled as supporters or fans. P10 (male, 25, White) revealed, "My favorite people, fans I guess, also watch [other streams] with all of the favorite fans of all of the other gay streamers. And so you kind of get this best of everybody uh feeling and it's kind of cool." For P10, considering viewers as fans was an effective way to gather people of similar interests together, leading to a positive social experience both for him and for the fans.

Especially, these supporters displayed loyalty, becoming people whom the streamers could count on through ups and downs. P13 (female, 31, White) shared her story: "I have like a group of people that have just have found me along the way and come and hangout and watch me no matter what I am playing." In this sense, viewers have also become a source of emotional support that sustains streamers' practices.

4.1.3 Social Grouping. For streamers who identified as part of a particular social group, such as LGBTQ identity or race, they expected that their audience would be of the same group. Attraction of people from these groups came from advertising their stream in other online communities that cater to their groups of interest. For example, P9 (male, 26, White) described how they promoted their stream to specific online groups: "The groups I advertise to are usually trans inclusive. I refuse to work with anyone who isn't, so usually the user base picks up on that."

According to P9, it would be important to convey such social identity to viewers so as to target the appropriate audience. Therefore, having tags added to their stream became a common practice - so that people who search for a specific tag would be able to find the streamers and affiliated themselves with that social group. P10 (male, 25, White), who had two streams - his personal channel and one that he did with other gay streamers under the banner of being a gay stream - further explained this situation. He claims that his two channels attract very different audiences. His personal channel consists more of local people, friends, and co-workers, while the group channel brings more of a queer audience.

Similarly, P16 (male, 37, Black) said that he was part of a number of Discord communities specifically for streamers of color. Many of his viewers were also affiliated with those communities. Because the streamers were not online $24 / 7$, they also helped each other out by directing their viewers to watch other streamers of color through "raids." Raids are a Twitch specific activity that describes when one streamer sends their viewers to another streamer's channels and the visitors bombard the chat with emoticons to show the surge of incoming viewers. When this happens, the streamer being "raided" would know that the other streamer was supporting them.

In addition to tags and raids, the discussions that happened with viewers also provided streamers with information about their viewers' identities. P4 (male, 34, White) noted, "identity is something that 
comes up in my channel right the discussions around um particularly LGBT stuff... we talk about race and cultural stuff in my channel too... so yeah $i$ would say $i$ would probably have a general anecdotal like a better idea of the sorts of people." Therefore, it seems to be important for streamers to have "an idea of the sorts of people" in their audience, especially when their streaming content related to certain social identity (e.g., "particularly LGBT stuff").

4.1.4 People Interested in the Content. P2 (male, 20s, Hispanic), who streamed art, said that he perceived most of his viewers as people who had some interest in art. This included those who were finishing high school and wanted to know more about the lifestyle of artists, other artists, or art enthusiasts. He explained," I do a lot of fan art. So I get a lot of gamers who are just fans of the games, who are just excited to see their characters imagined in other perspectives, imagined in silly costumes or in [other] various ways."

In P2's opinion, viewers came to his channel mainly because of the content. Streamers could infer that their viewers would be of a particular age because they were interested in certain content. This inference was often quite specific and not based on any data of all of their viewers. For example, P17 (male, 34, White), who streams board games, said that he assumed his viewers would be in their mid thirties to fifties: "I don't think we expect children or teens to really watch... they have so many other things they are probably interested in than board games."

P17's assumption of his viewers was grounded on his personal perception of typical board game players rather than any other cues. In contrast, some streamers, who had active presence on other social media such as Facebook or Twitter said that they were able to collect more demographic information from those sites. However, because Twitch did not provide any personal information about viewers, they had no other option than to guess - some streamers were better than others at pinpointing their viewer demographic.

For example, P3 (female, 41, White), who streams herself making quilts that she also sells online, was able to clearly identify the different types of viewers who watched her channel. She explained her rationale, "So, I get the people who are the 25 to 40 year old middle income that may have a house that are watching because they are like, 'Oh! That'll be cool to have a quilt like that.' Then on the other hand, I get the people that sew, that wanna learn to sew, that are in here learning about all the tips and tricks that I do. So, I have two completely different people who watch me." Yet P5 (female, 25, White) only had a vague idea of her viewers' demographics. "As I play a lot of Pokemon, I definitely have a younger audience. Ummm but it's hard to estimate," she said. In both examples, P3 and P5 had to "guess" the demographics of their viewers only based on the content that they streamed (e.g., making quilts and gaming). This can be challenging because some content attract a wider range of viewers than others.

Guessing who the audience are is even more challenging for streamers who broadcast a variety of different content, as P13 (female, 31, White) mentioned, "it's interesting how much the crowds change depending on what you're streaming. You kind of get a grab bag if it's a newer game or if it's like a DnD or something like that. That's a little more community based rather than game based itself. Then you get a lot of people that tend to be more helpful and want to share information and ideas and that's kind of the basis of that type of community for those kinds of games." Therefore, for such steamers, they were highly aware that their viewership would fluctuate depending on the content - whether that be different games or different content categories altogether.

4.1.5 Trolls. The term "troll" was used by streamers as a blanket term for people who engaged in any kind of deviant behavior. Streamers did not seem to differentiate trolls based on behavior type, even if they were acutely aware of the variety of harassment methods. P13 (female, 31, White) shared her experience of having trolls as part of the audience: "you do tend to get a lot of very basic kinds of trolls, ones that will show up and asks for nudes, or any pictures, anything like that or harass your gameplay, just in general because I am a female. So I mean, that's kind of expected, it's one of things where should it be like that?! Absolutely not. Is it and you have to deal with it? Yup that's kind of the world that we're in."

In P13's account, streamers seemed to accept trolls as an inevitable but undesirable reality of the current live streaming landscape. Obviously, they did not welcome trolls as their audience. However, there appeared to be consensus that trolls existed and were "kind of expected" as a type of common audience in their streaming practices. While they could not stop trolls from coming to their streams, they considered dealing with trolls an important aspect of audience management.

4.1.6 Lurkers. The final type of audience that streamers kept in mind during their streaming was lurkers. They conceptualized lurkers as viewers who were watching the stream but not participating in the chat, as P23 defined, "There are people who may be watching that never interact. A lot of people call them lurkers. I try to find the good balance between you know interactively acknowledging all the regulars subscribers but also acknowledging that there are people that could be lurking... and maybe they don't want to interact maybe they just want to see how the stream is." For smaller streamers, they were able to have a rough estimate of the percentage of lurkers by comparing the overall live viewer count with how many people they noticed in chat. For larger streamers, they were uncertain of the exact percentage, but assumed that a significant portion of the viewers would be lurking.

However, one of the main challenges with lurkers was that streamers did not know if the lurkers were unable to interact for various reasons (e.g., being at work, having the stream in the background) or if they were purposefully choosing not to interact. To address this challenge, P24 (female, 30, Hispanic) shared a clever method of trying to acknowledge lurkers who wanted to be noticed: "I will say something like 'okay everybody put an emote in chat and I'll read off your name and say hi.' Even if you are lurking and you don't want to say anything, you can just type this once and I will try to go through the list quickly.' It's usually pretty comical trying to read all the names off really fast. In a way, this involves the lurkers a little bit without them having to talk too much. They can say hi and get a hello back and then that will be it." P24's strategy was proactive. Instead of waiting for lurkers to participate, she proposed some simple activities to engage them. This proactive approach also helped her better perceive various types of lurkers and identified those who would actually be willing to be more interactive. 


\subsection{Audience Discovery}

RQ2 inquired into how streamers figure out who their perceived audience is. From our data, we identified three ways through which streamers discovered their audiences: talking on and off Twitch as well as using internal and external tools.

4.2.1 Explicit Questioning - On and Off Twitch. The easiest way to learn about who is watching at the moment is of course, by asking people. Conversing with the audience on chat enabled streamers to learn about who is present, especially when the pace of chat is slow enough that the streamers could read, process, and respond.

In doing so, sometimes streamers outright asked their viewers who was present, as P12 (female, 31, Pacific Islander) did: "I get replies from them like 'Oh it's 8 am in Australia,' or 'Hi from UK and it's 4 here.' So this is how I come to know that where are my viewers located." In addition, demographics were another type of information that viewers were oftentimes willing to share. P20 (male, 29, N/A) shared, "there have been conversations about age, or life, or if somebody's like, 'I have two kids.' It's very easy to tell that they're much older. A common question is, people ask me how old I am, and I'll them I'm almost 30. They'll be like, 'Wow, you look a lot younger.' Or, 'I would have never guessed.' So then people on chat will mention their age, and how I look young, which I guess is a compliment." Based on these quotes, asking and sharing demographics such as location and age seemed to be an effective way to help streamers and viewers get to know each other and start conversations.

Another way of learning more about their viewers was to ask questions such as "What did you guys do today?" and letting people respond in the comments. P19 (female, N/A, White) further described how this learning process was no different than how people learn about others in offline situations - starting with casual conversations that ask questions about each other that eventually build up into relationships.

Since streaming is not a one-off event, streamers would also learn more about their viewers via triangulation of data across multiple social media platforms and remembering their Twitch user ID. For example, P4 (male, 34, White) mentioned, "I keep chat open when I stream and I can see who is talking and who is engaging with the content. I can find out more information about them because people engage with the content afterwards, leave comments on Youtube, Tweet at me, engage in conservation on forums that kind of thing." Similarly, P8 (male, N/A, mixed) added, "you can know them by their tags online, by their names in chat. You can tell them apart from their personalities and stuff. You can get a lot across from how they talk and things they say and what they're trying to do and things they're looking for." According to $\mathrm{P} 4$ and P8, it was common for streamers to rely on multiple methods, cues, and channels out of Twitch to better discover and understand their audience. For example, their presence on Youtube or Twitter and the content they shared on other platforms could all provide streamers with useful information about their audiences' interests and personalities.

In addition, Discord was a popular communication application that many streamers seemed to be using as a back channel for more intimate conversations. P2 (male, 20s, Hispanic) said that he encouraged his viewers to hang out with him on Discord and the conversations held there informs him more about who they are and their lifestyles. This was a common sentiment echoed by others, such as P6 (female, 19, Asian): "it is pretty amazing to see all the fans come together like that. Since they're in my Discord server, I get to know them a little bit more better as an individual person, like their lives, what they do, what they are studying, things like that. So, when I see them in stream, I'm talking to someone who is like an actual friend. I've gotten to know them and they are not just random viewer99." In P6's opinion, communication applications out of the streaming platform seemed to provide streamers with open and supplementary spaces to interact with viewers. Such interaction was highly important for her to better discover and understand her audience - as "an individual person" or "an actual friend" rather than "just random viewer99."

4.2.2 Aggregated Information from Internal Tools. Internal tools are functions and system features that are provided by Twitch. These include both analytical tools and other features that streamers repurpose for analytical purposes.

For example, the Analytics provided by Twitch is aggregate data that can offer streamers a general idea of viewer engagement at a very high level. This feature was added by Twitch in recent years and was available on a dashboard where streamers could see metrics related to their channel. P20 (male, 29, N/A) explained how he used the Twitch Analytics to understand his audience, " Twitch will provide them to you post-stream. Sometimes it takes about 10 to 15 minutes for those analytics to come in. But it'll tell you how long you streamed, who your average viewers were, what your max viewers were, how many new follows you got, how many unique people talked in your stream. That's kind of the data that I pull from it." For him, these analytics seemed to offer a valuable summary of audience involvement as a post-stream reflection.

Some other tools are not necessarily analytical tools, but informational features that can help with streamers' sense-making of their audience. P2 (male, 20s, Hispanic) shared an example, "there is a button you can hit from the streamer side on Twitch, which is near your chat window. It shows you the current viewer and moderator list in your channel. So, you can see who they are, who is working on Twitch, who is just there but not chatting. You can see if you have any moderators in the room something like that." According to P2, in addition to knowing regular viewers, identifying whether or not moderator was part of the audience was also an important practice (e.g., for helping managing audience and preventing trolling/harassment), which could be achieved by using the built-in button.

Aside from being able to identify whether moderators are watching, the ability to view the list of everyone in chat was primarily used by smaller streamers when they wanted to see if they recognized "regulars" who came to their channel. However, the analytics could sometimes be a burden to them - an indicator of failure. P19 (female, N/A, White) shared, "Sometimes, yeah, I try to not look at anything relating or similar to my viewer counts because if I am having like a low viewer count day that can bum me out. That's kind of just a common thing among all streamers having low numbers, got a bum you out. So I kind of stay away from that because I want to focus on keeping everybody in my chat happy and feeling well." For smaller streamers like P19, they were in fact reluctant to look at any statistics related to viewer traffic because they did not want to be disappointed. 
Some other streamers, however, chose not to engage in realtime sense-making and opted to wait until after the stream. P21 explained: "it can be distracting if I'm looking through my viewer list. I try not to look at those kinds of things when I stream. What I would do is after the stream I will definitely look at my statistics, and see how I'm getting traffic into my stream. Where they're coming from whether it's Google, or whatever." According to P21, trying to use the list feature to look at who was in the stream was a time consuming task that distracted them from their content.

4.2.3 Detailed Information from External Tools. Very few streamers used external tools to help them manage audience. These streamers tended to be a little more technologically savvy and interested in analytics that are not provided within Twitch.

For example, P3 (female, 41, White) said that there were some third party software that gives one more detailed information than the Twitch-provided metrics about followers, subscribers, and other information on a daily, weekly, and monthly basis. In this way, she learned about these tools while networking with other streamers at TwitchCon.

In addition, P8 (male, N/A, mixed) said that he used analytics from other social media, such as statistics from their YouTube channel, Instagram, and Twitter. These tools, however, did not convey real-time information or information about any particular individual. Therefore, streamers would usually look at this information before or after their streaming session. Moreover, the information about viewers did not include any demographics of the viewers. Streamers would still rely on other approaches of informationseeking such as the conversational methods mentioned above.

\subsection{Methods of Managing Interactions with Different Audiences}

Most streamers noted that their audience was "everyone" because interaction with viewers was a core feature of streaming. This was much easier for those who only had a small group of viewers. Yet even for streamers who had hundreds or thousands of viewers or more, they considered it a social responsibility/pressure to interact with as many people as possible, as viewers would sometimes complain or be sad when streamers did not directly respond to their questions or donations.

As an effort to interact with "everyone," streamers tended to conceptualize their audience into two types: an abstract audience of unknowns, or a specific person. Balancing the abstract audience with specific persons was one of the most significant challenges that all streamers seemed to be extremely mindful of. In addition, technological challenges were brought up as well since they discouraged some streamers to directly address specific viewers due to time-lag in the streaming. This delay may often confusions, as P6 (female, 19, Asian) said, "I try to keep my statement or whatever I do pretty open to everyone who is viewing. Specially because Twitch has the stream delay between whatever I do and the chat."

P6's explanation illustrates that despite the concept of live streaming as being a synchronous medium, the slight delay between the broadcasting and when viewers actually see it can lead to major communication issues. Even if streamers respond immediately to a viewer when they see his/her comment, it would still be a few seconds (or sometimes more) after the viewer posted the comment.
In this situation, if the streamer responds to a specific person, the response may seem to be out of context because the chat has moved on quickly. This was particularly an issue for streamers whose viewers were very active in chat.

In this section, we present five main methods that streamers used to manage their interactions with different audiences.

4.3.1 Balancing General Audience with Individuals. Sometimes, streamers only had a vague idea that they were dealing with "viewers" in general. In order to cater to this abstract audience, a streamer often would talk about very generic topic that everyone could engage in but he or she did not have to respond to each person. Some common go-to topics that streamers mentioned were the weather, sports, what they had for their latest meal, or the generic "How's everyone doing today?" question. These topics usually elicited responses from the viewers and engaged them without having to single out a particular viewer. Of course, streamers did not consider these topics very meaningful. However, such mundane conversations could become an effective way for streamers to know and manage interactions with their viewers. This could be especially valuable for streamers who streamed frequently and regularly.

While most streamers had both an abstract audience and specific people in mind, five streamers said that they purposefully endeavored to focus on the larger group and tried to minimize individual interactions as much as possible. They explained various reasons for limiting interactions at a group level. One of the most important reason was due to the overwhelming nature of group chat, as $\mathrm{P} 4$ (male, 34, White) mentioned, "I try not to respond directly to individuals because that that can get very overwhelming very quickly depending on the size of the conversation. What I often try to do is to engage in the overall conversation, be aware of what the conversation looks like, what people are talking about, and then address points that are coming. If someone makes a really good point or says something that I want to boost as a message, then I will engage with the specific point. But I would say more generally I kinda follow the conversation rather than engaging directly with the individuals. I am careful with that because that person hasn't necessarily volunteered to be the focus of everyone's attention." According to $\mathrm{P} 4$, following the overall trend of the conversation, or focusing on the bigger picture of the chat, was crucial to manage interacting with a sizable group of audience. In this way, it was easier to control the general direction of the conversation without getting lost/distracted or imposing unwanted attention to a specific viewer.

In contrast, some other streamers focused on thinking of a specific viewer when they were interacting with their audience. They highlighted two main reasons for doing so. The first was to address a specific point or topic that an individual said in chat. The second was because they had trouble conceptualizing their audience as an abstract group, saying that they never think about it as a "collective"(P5, female, 25, White) or "amorphous mass" (P15, female, 52, White).

When it came to deciding exactly when to "zoom in" to a specific person or "zoom out" to a group conversation, streamers also had different criteria for what they thought would be a topic or person warranting more attention. When it came to interacting with a specific viewer, most of the time the viewers who caught streamers' attention were those who frequently visited the stream. Streamers 
pointed out that they were able to identify dedicated viewers immediately because of the familiarity of their username. P20 (male, 29, N/A) also added how this strategy may encourage long-term engagement: "I think for me, it's making sure that they have a good first impression to give them a reason to come back. So if they're a new follower... Let's say, Dusty. It's like, "Oh Dusty, thank you for the follow. I really appreciate it. I hope you're doing well. If you have any questions or anything, let me know." And not that I say that every time for every fellow, but I'll try to make it a little personal. [...] And I know that goes miles with people." In P20's example, paying attention to and interacting with a specific viewer was a necessary way to acknowledge and show appreciation to new followers. Such special attention would not be conveyed by just delivering generalized information to everyone.

In summary, streamers discussed how balancing between a general, abstract audience and interacting with a specific person was very complicated. P4 (male, 34, White) shared his experience: "I would say that when I am engaging in chat on and off, I am aware of the qualities of any given individual. But I am also managing the conversations as a whole, the chat as a subdivided entity having a conversation either with me or with each other." P13 (female, 31, White) also added, "I have a degree in public speaking so I've trained in a lot of professional situations and stuff like that before. So I kind of have a fluid ability to single out somebody and have a little bit of conversation with just them and be able to and address the entire group again as well. I do address them both as just like "hey guys," or something like one would address in an audience entity and be able to talk to a couple of people at the same time. I try to greet everyone individually and by name as people speak to me and as they come into the channel. It's just a matter of giving individuals attention and being able to really talk to them as a whole. Based on these quotes, such complexity seemed to situate in the fact of less of toggling between different audiences and more of conceptualizing the audience as a hybrid audience through live streaming.

\subsubsection{Brute Force Method to Engage With All, But at the Cost of} Content Creation. Streamers, especially smaller ones who did not have much chat volume employed a "brute force" method of trying to process all information as it came. This included responding to all viewers' comments and sometimes even taking a break from the streaming content to interact with everyone. For example, P15 (female, 52, White) noted, "for me right now, that's pretty easy. I can pretty much respond to every comment because I have not a ton of viewers at the time. On the time when I was getting more viewers, when I was actually doing that ill-fated late 2016 push, I did find that I'd have to pause periodically to go through and scan the comments. I would answer questions and would respond to things that I thought were needing response. In those situations, I would tend to say, "Well, I'm looking over a chat now and catching up." According to P15, while it was beneficial and encouraging to respond to everyone, it was only possible for streamers to employ this method because there was not a lot of chat activity.

Oftentimes, this brute force method would require taking time off of content creation in order to address all the chat comments, as P10 (male, 25, White) shared: "if there's a bunch of people in chat I try to answer everyone, I even take pauses in my game and go back to make sure I don't miss anything in chat and communicate with people so they don't think I'm ignoring them or think I'm favoring other viewers." Based on P10's account, streamers had to constantly evaluate the need to respond to all comments and the desire for created content due to the limited time that they had to manage both of them. In this process, balancing or even sacrificing one aspect over the other to some degree seemed to be inevitable.

4.3.3 Prioritizing Certain People or Topics. Despite streamers' efforts at trying to interact with as many people as possible, the realistic limitations forced them to prioritize some viewers over others, even if there was not a concerted effort to do so.

Many streamers talked about how subscribers were a priority for them. Subscribers are those who pay a monthly subscription fee, of which the streamer receives a share. Aside from the monetary reasons, however, the system affordances also make it easier to identify subscribers because they have "badges" next to their user name: "there is a division between who obviously is subscribed to me and who isn't. Because the system is encouraged it encourages us to be able to engage in that differentiator," said $\mathrm{P} 4$.

In addition, there was also a difference between prioritizing people versus prioritizing different types of content. P16 (male, 37, Black) explained, "I don't respond to everything, and especially if I'm really wrapped in the story, or like if I'm at kind of like a critical juncture in the game, I'd definitely sort of prize questions over responding to every single comment." According to P16, he would usually respond to any question but did not feel obliged to address standalone comments. One reason would be that streamers looked to engage more comments that naturally elicited further conversation.

In a different example, P5 (female, 25, White) discussed how she usually responded to the short comments first because they were faster to read and oftentimes easier to respond to: "I sometimes skip very long messages and decide to read them when I am free because it'll take longer to read and to respond to that. So I guess it's some sort of efficiency thing." Though P16 and P5 used different criteria to prioritize whom he/she would respond first, a consensus seemed to focus on comments or topics that would effectively encourage more interactions and chat activities.

4.3.4 Relying Upon Moderator Help to Overcome Cognitive, Design, and Temporal Limitations. If streamers missed a comment or failed to answer a viewer's questions, sometimes the moderators would point this out to the streamer. Streamers discussed how they scanned the chat for visual indicators for their moderators' green badge that would appear next to their IDs. For example, P3 (female, 41, White) revealed, "I try to respond to as much as I can, to everyone. I finally have a couple of mods that are good and if I miss something they make sure, so anytime I have a new follower, a new sub anything like that... if I am not on that right away they are in there and they say something and so I scan for those little [mod] badges just to make sure that ok, I am not missing anything, am I? Because sometimes the chat does go by pretty fast." As streamers had to navigate through different dimensions of streaming (e.g., creating content, performance in front of the camera, and monitoring audience), it would be challenging and cognitively exhausting for them to follow up with every viewer. In this sense, moderators' presence and help was critical for the streamers to keep track of viewers' comments and respond. 
In a unique example, one streamer sought the labor of moderators to read chat to him while he focused on creating content. P2 (male, 20s, Hispanic), an artist who creates digital illustrations, shared his story:"on my stream, I have two monitors and a drawing monitor, which I'm drawing on and the entire screen is occupied by the drawing space so there's no other secondary windows on there that I can preview the chat. If I'm drawing or really focusing rendering something making it look right, it requires most of my focus. So early on I asked [him] if he would be willing to help me and just read the chat to me sometimes or letting me know somebody was speaking to me directly. It helps me filter out when viewers are talking to each other or discussing things amongst themselves." P2's case is interesting because it was difficult for him to be drawing and reading chat at the same time. However, he valued interactivity. To address this challenge, his two moderators - one of whom was his fiancé- also participated in stream through audio. They read the chat aloud to him or set aside messages that they thought he (as the streamer) should read to the audience. This strategy, therefore, effectively helped him balance between content creation and interacting with audience.

4.3.5 Randomly Engage - Last Resort, When All Else Fails. We call the last method that streamers as "random" because there was no systemic method or thought process that went into their interactions. The streamers had limited attention to engage with the users systematically or had not discovered systematic method that satisfied them. For example, one of them suggested that whatever chat they happened to read at the time was what they decided to respond to: "It just comes down to who is catching my eye first basically," described P5 (female, 25, White). This "random" notion was seen as the last refuge to manage the cognitive overload from constantly refreshed chat during content streaming. It also reflects on how the current design of streaming platforms lacks sufficient scaffolding for the streamers to best discern and engage with their audience.

\section{DISCUSSION}

Using our findings as a basis, we now discuss the implications of this work for extending our current understandings of audience management in online social spaces as well as potential design directions that may support audience management in live streaming.

\subsection{The Uniqueness of Managing Audiences on Twitch}

Existing studies on audience management often tend to focus on social media platforms (e.g., Facebook, Google+, Twitter, and Reddit) $[4,18,19,21,24,25,27]$. In this paper, we build upon this existing work on sociotechnical systems and endeavor to investigate how studying a unique and novel form of sociotechincal system (e.g., live streaming) may bring in new insights on audience management in online communities.

As we have described at the beginning of this paper, Twitch, along with other similar live streaming platforms, are quite different from social media platforms. Live streaming combines public broadcast of high fidelity live audio and video through Internet and low fidelity shared text-based channels open to both streamers and viewers [13]. This complex media ecology also leads to the complicated mechanisms through which streamers interact with a large group of diverse viewers, including both maintaining a general audience and making appropriate impressions to specific audience.

Despite the unique technological affordances of live streaming platforms, our findings still confirm that imagining and categorizing audience is considered to be important and is performed for successful audience management in any type of sociotechincal systems. For example, our data show that to prepare themselves for the streaming practices, streamers may still tend to construct a general conceptualization of whom may watch their streaming (i.e., the imagined audience; see $[24,25])$, though they might use various information and social cues from the streaming platform to construct such a conceptualization (e.g, the perceived demographics or topical interests). Our findings also reveal six categories of viewers depending on the social meanings that viewers hold to streamers as well as streamers' perceptions of viewer characteristics, which is consistent with the criteria of life facets and topical interests to manage contacts as Kairam et al. [18] show.

We point to the importance of conceptualizing audience management as a multi-agent mechanism. In previous studies, audience management places much agency on the performer: controlling what to share, how to share, and whom to share/interact with is often the user's decision alone; and such controlling is usually accomplished by either creating multiple profiles/accounts [10,21] or setting up multi-level access/privacy limitations [10,18]. What we see from our data is that audience management becomes a multi-agent mechanism on Twitch: it is beyond the streamer's own decision-making or the system affordances alone. Rather, managing audience on live streaming involves multiple parties: the streamers, his/her moderator(s), and even the audience themselves. We have seen how streamers heavily depended on moderators' voluntary labor to identity and interact with audience strategically (e.g., a reminder from the moderator to answer a viewer's questions); they also closely communicated with their moderators at the back stage (e.g., on a private chat or third party communication tool) to collaboratively refine how they should interact with different audiences. We have also seen moderators directly participated in the front stage - streamers' actual interactions with different audiences (e.g., the moderator joined the audio chat with viewers). In addition, our data shows that streamers strategized their interactions with audiences based on their prior experiences or impressions of their audience base (e.g, people of a particular age). In this sense, their audiences in the past also affected how they managed and interacted with the current audiences.

Furthermore, the nature of live streaming suggests the challenge to manage different audiences in traditional ways such as using multiple accounts of multi-level privacy setting. It is crucial for streamers to maintain consistent and well-established identities/accounts so they can attract a stable audience base. There is also no access limitation settings on Twitch as it is a public platform for openly sharing all streaming content. Instead, streamers tend to creatively use a combination of multiple internal (e.g., real-time signals provided by Twitch) and external tools (e.g., third party software) as sources of information for their audience management practices. As a result, strategies and techniques to manage audiences on live streaming tend to be lesser constrained by the system itself but more personalized and diversified. 


\subsection{Design for Managing Audience in Livestreaming}

Our findings describe the perceptions of audience from a streamer's perspective. These perceptions are guided by a strong individualistic agency by a streamer who has to expend significant bandwidth to enquire the viewership details. This work is performed along and in aid to the "primary" job of content conceptualization, design, production, presentation, and post-production. However, lack of audience and viewership information affords little guidance to a streamer to engage more inclusively with them, and nurture content appropriately. It is clear that these streamers go through great lengths to engage in information seeking behaviors akin to information "foraging" [30]. Once they have this information, however, there are no tools that can help them document their findings. Thus we have identified the following design directions:

5.2.1 Audience Feedback Mechanisms. The current streaming platforms enable engagement by audience during streaming via text/chat responses. However, managing these responses is costly (cognitively, and temporally). Further, while the streamer continues to share their performance as an outgoing stream, they receive limited feedback from their audience- unlike a traditional theatrical performance, where a performer can scan the audience to get a somewhat cursory perception of demographics, or responses to their craft $[5,36]$, the current streaming platforms do not afford such benefits to the streamers. Plus, currently the platforms offer a somewhat imbalanced engagement model where streamers have to continuously engage, enquire, and share more information, while the audience members have limited choices. Of note, this research was conducted before Twitch introduced its Channel Points feature, which let streamers run polls, but there are still limited ways that audience members can signal their attention or sentiments aside from emojis, and even these methods are restricted from those who choose to post things in chat. Future tools that allow the audience to give one-click types of feedback [14] may benefit the streamers as these lightweight feedback mechanisms have been shown to provide the recipient with a sense of social support [8, 38].

5.2.2 Real-Time Implicit Aggregated Audience Statistics. Presently, the streamers have to query their viewers for demographics, and interests. Some users, when queried, respond with their details. These explicit requests are hard to manage "by brute force" as the responses fly by fast, and streamers are partially distracted by the content production. On the other hand, such details can be offered as aggregates to the streamers in real-time (for example, an info graphic) without an explicit request. This helps manage streamer's attention, while providing information a streamer might find useful for content production and audience engagement.

5.2.3 Visual Cues of Audience Categories. As the streamers mentioned multiple times, they do not view the entire audience as a monolithic block. They have categorized their viewing into multiple semantic categories like family, supporters, lurkers, trolls, etc. Presence of such information enables the streamers to not rely on their memory when engaging with an audience member, for example when answering questions by an audience member, it would be useful to know if this is a lurker who has engaged for the first time. We recommend providing visual cues such as labels on users that only the streamer can see, for easier identification of the audience members as they engage. Having such contextual information will enable streamer to decide how, how much, and when to engage.

5.2.4 Engagement History. As the audience grows for a streamer, it may become challenging to continue to remember, nurture and engage with new members. This not only requires attention away from the content, but also requires memory recognition of an audience member and recalling their last engagement. By providing past engagement history relevant to the audience members, a streamer may be better equipped to engage in a customized way with each member. Currently, viewers who pay (i.e., subscribers) have a badge that indicates how long they have subscribed [40], but there are should be more diverse methods of discerning different types of engagement for both paying and non-paying audience members.

\section{LIMITATIONS}

This work has primarily focused on Twitch as the streaming platform, to recruit streamers from and consequently discuss implications for Twitch's design. Multiple other platforms exist for streamers, like YouTube, Mixer, or YY (Chinese) that have some similarities but have not been the focus of this work. Future works should consider focusing on other platforms to identify common challenges and strategies across the different platforms and explore potential cultural differences. The users participating in this research reflect a convenience sample of primarily Western users who were willing to share their experiences and were mostly recruited through TwitchCon and (English) Twitter. These streamers were more likely to have stronger streamer identities than the average streamer.

This study aimed at looking at more general patterns of audience management, but there are also more nuanced interactions for specific scenarios, such as moderation related to dealing with toxic viewers or paying vs. non-paying viewers. Future studies may want to explore unique contexts and/or scenarios.

While a sample of 25 should overcome some problems inherent to convenient sampling, we also did not discover any significant differences based on gender or sexual orientation within our sample. However, we recommend future researchers to pursue broader recruitment as such lines of enquiry were not our primary focus.

\section{CONCLUSIONS}

In this research, we found that streamers have mentally segmented their audience into groups and used this grouping to help with splitsecond decision making, which needs to take place in the real-time live streaming environment. In doing so, They used both technical and social methods to find out about their viewers prior to the mental sorting, and also as an effective approach to help manage their interactions with diverse audiences. The layered and dynamic audience management practices that we identified in the live streaming context indicates that self presentation theories that simplify the agents into performer and audience require more nuance in this new media landscape and that the human and computer-assisted interactions reflect the complexity of newer sociotechnical systems.

\section{ACKNOWLEDGMENTS}

We thank our participants and reviewers. This work is supported in part by the National Science Foundation awards \#1841354, \#1849718. 


\section{REFERENCES}

[1] 2017. The State of Live Streaming - Statistics and Trends. https://www.goglobe.com/blog/live-streaming-statistics

[2] 2019. Techjury. https://techjury.net

[3] 2019. Twitch Tracker. www.twitchtracker.com

[4] Jane Bailey, Valerie Steeves, Jacquelyn Burkell, and Priscilla Regan. 2013. Negotiating with gender stereotypes on social networking sites: From "bicycle face" to Facebook. Fournal of Communication Inquiry 37, 2 (2013), 91-112.

[5] Elizabeth Bell. 2008. Theories of performance. Sage.

[6] Jie Cai and Donghee Yvette Wohn. 2019. Live streaming commerce: Uses and gratifications approach to understanding consumers' motivations. In Proceedings of the 52nd Hawaii International Conference on System Sciences.

[7] Jie Cai, Donghee Yvette Wohn, Ankit Mittal, and Dhanush Sureshbabu. 2018 Utilitarian and hedonic motivations for live streaming shopping. In Proceedings of the 2018 ACM international conference on interactive experiences for TV and online video. ACM, 81-88.

[8] Caleb T Carr, D Yvette Wohn, and Rebecca A Hayes. 2016. As social support: Relational closeness, automaticity, and interpreting social support from paralinguistic digital affordances in social media. Computers in Human Behavior 62 (2016), 385-393.

[9] Mia Consalvo and Andrew Phelps. 2019. Performing game development live on Twitch. In Proceedings of the 52nd Hawaii International Conference on System Sciences.

[10] Michael A DeVito, Jeremy Birnholtz, and Jeffery T Hancock. 2017. Platforms, people, and perception: Using affordances to understand self-presentation on social media. In Proceedings of the 2017 ACM Conference on Computer Supported Cooperative Work and Social Computing. ACM, 740-754.

[11] Erving Goffman et al. 1978. The presentation of self in everyday life. Harmondsworth London.

[12] Oliver L Haimson and John C Tang. 2017. What makes live events engaging on Facebook Live, Periscope, and Snapchat. In Proceedings of the 2017 CHI conference on human factors in computing systems. ACM, 48-60.

[13] William A Hamilton, Oliver Garretson, and Andruid Kerne. 2014. Streaming on twitch: fostering participatory communities of play within live mixed media. In Proceedings of the 32nd annual ACM conference on Human factors in computing systems. ACM, 1315-1324.

[14] Rebecca A Hayes, Caleb T Carr, and Donghee Yvette Wohn. 2016. One click, many meanings: Interpreting paralinguistic digital affordances in social media. Journal of Broadcasting \& Electronic Media 60, 1 (2016), 171-187.

[15] Zorah Hilvert-Bruce, James T Neill, Max Sjöblom, and Juho Hamari. 2018. Social motivations of live-streaming viewer engagement on Twitch. Computers in Human Behavior 84 (2018), 58-67.

[16] Bernie Hogan. 2010. The presentation of self in the age of social media: Distinguishing performances and exhibitions online. Bulletin of Science, Technology \& Society 30, 6 (2010), 377-386.

[17] Mu Hu, Mingli Zhang, and Yu Wang. 2017. Why do audiences choose to keep watching on live video streaming platforms? An explanation of dual identification framework. Computers in Human Behavior 75 (2017), 594-606.

[18] Sanjay Kairam, Mike Brzozowski, David Huffaker, and Ed Chi. 2012. Talking in circles: selective sharing in google+. In Proceedings of the SIGCHI conference on human factors in computing systems. ACM, 1065-1074.

[19] Sanja Kapidzic and Susan C Herring. 2015. Race, gender, and self-presentation in teen profile photographs. New Media \& Society 17, 6 (2015), 958-976.

[20] Jina Kim, Kunwoo Bae, Eunil Park, and Angel P del Pobil. 2019. Who will Subscribe to My Streaming Channel? The Case of Twitch. In Conference Companion Publication of the 2019 on Computer Supported Cooperative Work and Social Computing. 247-251.

[21] Alex Leavitt. 2015. This is a throwaway account: Temporary technical identities and perceptions of anonymity in a massive online community. In Proceedings of the 18th ACM Conference on Computer Supported Cooperative Work \& Social Computing. ACM, 317-327.

[22] Pascal Lessel, Alexander Vielhauer, and Antonio Krüger. 2017. Expanding video game live-streams with enhanced communication channels: a case study. In Proceedings of the 2017 CHI Conference on Human Factors in Computing Systems. ACM, 1571-1576.

[23] Jie Li, Xinning Gui, Yubo Kou, and Yukun Li. 2019. Live Streaming as CoPerformance: Dynamics between Center and Periphery in Theatrical Engagement Proceedings of the ACM on Human-Computer Interaction 3, CSCW (2019), 1-22.

[24] Eden Litt. 2012. Knock, knock. Who's there? The imagined audience. Fournal of broadcasting \& electronic media 56, 3 (2012), 330-345.

[25] Eden Litt and Eszter Hargittai. 2016. The imagined audience on social network sites. Social Media+ Society 2, 1 (2016), 2056305116633482.

[26] Zhicong Lu, Haijun Xia, Seongkook Heo, and Daniel Wigdor. 2018. You watch, you give, and you engage: a study of live streaming practices in China. In Proceedings of the 2018 CHI conference on human factors in computing systems. ACM, 466.
[27] Elliot T Panek, Yioryos Nardis, and Sara Konrath. 2013. Defining social networking sites and measuring their use: How narcissists differ in their use of Facebook and Twitter. Computers in Human Behavior 29, 5 (2013), 2004-2012.

[28] Anthony J Pellicone and June Ahn. 2017. The Game of Performing Play: Understanding streaming as cultural production. In Proceedings of the 2017 CHI Conference on Human Factors in Computing Systems. ACM, 4863-4874.

[29] Andrew Phelps and Mia Consalvo. 2020. Laboring Artists: Art Streaming on the Videogame Platform Twitch. In Proceedings of the 53rd Hawaii International Conference on System Sciences.

[30] Peter Pirolli and Stuart Card. 1999. Information foraging. Psychological review 106, 4 (1999), 643

[31] Howard Rheingold. 1993. The virtual community: Finding commection in a computerized world. Addison-Wesley Longman Publishing Co., Inc.

[32] Anselm L Strauss. 1987. Qualitative analysis for social scientists. Cambridge university press.

[33] Minhyang Suh, Frank Bentley, and Danielle Lottridge. 2018. " It's Kind of Boring Looking at Just the Face" How Teens Multitask During Mobile Videochat. Proceedings of the ACM on Human-Computer Interaction 2, CSCW (2018), 1-23.

[34] John C Tang, Gina Venolia, and Kori M Inkpen. 2016. Meerkat and periscope: I stream, you stream, apps stream for live streams. In Proceedings of the 2016 CHI conference on human factors in computing systems. ACM, 4770-4780.

[35] TL Taylor. 2018. Watch me play: Twitch and the rise of game live streaming. Princeton University Press.

[36] Mick Wallis and Simon Shepherd. 2018. Studying plays. Bloomsbury Publishing.

[37] Donghee Yvette Wohn. 2019. Volunteer moderators in twitch micro communities: How they get involved, the roles they play, and the emotional labor they experience. In Proceedings of the 2019 CHI Conference on Human Factors in Computing Systems. 1-13.

[38] Donghee Yvette Wohn, Caleb T Carr, and Rebecca A Hayes. 2016. How affective is a "Like"?: The effect of paralinguistic digital affordances on perceived social support. Cyberpsychology, Behavior, and Social Networking 19, 9 (2016), 562-566.

[39] Donghee Yvette Wohn, Guo Freeman, and Caitlin McLaughlin. 2018. Explaining viewers' emotional, instrumental, and financial support provision for live streamers. In Proceedings of the 2018 CHI conference on human factors in computing systems. ACM, 474.

[40] Donghee Yvette Wohn, Peter Jough, Peter Eskander, John Scott Siri, Masaho Shimobayashi, and Pradnya Desai. 2019. Understanding Digital Patronage: Why Do People Subscribe to Streamers on Twitch?. In Proceedings of the Annual Symposium on Computer-Human Interaction in Play. 99-110.

[41] Donghee Yvette Wohn and Cliff Lampe. 2018. Psychological Wellbeing as an Explanation of User Engagement in the Lifecycle of Online Community Participation. In Proceedings of the 2018 ACM Conference on Supporting Groupwork. ACM, 184-195.

[42] Jamie Woodcock and Mark R Johnson. 2019. The Affective Labor and Performance of Live Streaming on Twitch. tv. Television \& New Media (2019), 1527476419851077 . 